GANGER OF BONES.

By Group CAPTAIN STANFORD CADE, F.R.C.S., M.R.C.P., R.A.F.V.R.

(Surgeon to Westminster and Mount Vernon Hospitals; Consultant in Surgery to the Royal Air Force.)

Malignant tumours of bone can be classified into two main groups ; primary and secondary. The distinction is of clinical importance, as the treatment of metastatic tumours is naturally palliative in nature, whilst attempts at a permanent eradication of the disease should be made in the case of a primary growth. This contribution deals only with the primary tumours.

\title{
Primary Malignant Tumours of Bone.
}

The complexity of normal bone structure is reflected in the varieties of neoplasms derived from bone tissue. In general, the pathogenesis of a tumour depends upon the normal structure from which it is derived. In the case of bone, when reduced to its simplest concept, nerplasms may originate from bone cells, from bone marrow cells, from the endothelial elements of the vascular supply, from the adjoining fibrous tissue, and finally from some specific type of bone cell at some stage of its development. It is not surprising that the multiplicity of origin and the numerous variations related to the effect of the tumour on the parent bone should have led to elaborate classifications. For practical purposes, however, these can be simplified and, excluding sub-groups and variations in one and the same main group, five main types can be ecognised.

I. Osteogenic Sarcoma, derived from bone cells.

2. Ewing's Tumour, derived most probably from the endothelial cells of the bone marrow.

3. Multiple Myeloma, originating in the various cells of bone marrow.

4. Parosteal Sarcoma, an extraperiosteal tumour, falling into the group of fibrosarcoma derived from the outer layers of the periosteum.

5. In addition, a tumour, most often benign, but occasionally malignant, of obscure origin and of characteristic clinical and histological features-the osteoclastoma.

These five types of primary tumours of bone are distinct entities; their natural histories are quite different, the clinical features, the morbid anatomy, incidence, course and prognosis are distinct; they are, in fact, five different diseases occurring in the same structure-bone.

THE EFFEGT OF THE TUMOUR ON THE PARENT BONE.

The development and growth of a neoplasm within or in close apposition to bone leads to various changes in the osseous structure. The main changes fall into one of two categories : bone destruction and bone formation. At times both these processes proceed together, normal bone being destroyed and new bone being formed, although one or the other process is the predominant feature. These changes are plainly visible in radiograms and thus two main groups of tumours are distinguished : osteolytic and osteoblastic. The practical importance of this sub-division lies in the tendency of spontaneous fracture in the former type and in the differential diagnostic significance of the latter, according to the character and arrangement of the new bone. These changes are manifestations of the reaction of normal bone to the neoplastic processes.

\section{Osteogenic Sarcoma.}

\section{MAIN GLINIGAL FEATURES.}

It is chiefly a disease of the second decade ; most cases occur in patients between fifteen and twenty years of age. There is a slight preponderance in males. No bone is immune, but the commonest bone affected is the femur. In their order of frequency the following bones are involved : femur, tibia, humerus, innominate bone, fibula, scapula. Thus the lower limb and especially the region of the knee shows a marked preponderance of cases. The metaphysial end of the diaphysis is the common site of origin. Bone formation and bo ne destruction occur and according to the spread of the tumour and the effect on the par ent bone-periosteal and endosteal types are recognised (see below : Radiographic appearances). Vascularity is always marked, but in rapidly growing endosteal types the blood-vessels and sinusoidal blood spaces may be so numerous as to warrant the name of telangiectatic sarcoma. Histologically the tumour consists of spindle cells of various sizes in a matrix of fibrous, hyaline, cartilagenous, osteoid and osseous tissue. The main clinical features are pain and tumour. Pain always precedes the appearance of tumour. At first insidious and slight, it becomes more persistent and progressively increasing in intensity. The tumour is hard, usually fusiform 
in the long bones, but sometimes spherical or lobulated. The skin over it, in well-established lesions, is shiny and shows enlarged subcutaneous veins. Spontaneous fungation in untreated cases hardly ever occurs. Secondary local manifestations due to pain and the presence of a tumour, are muscular atrophy, synovial effusion in and disturbance of function of neighbouring joints. Metastatic tumours occur chiefly in the lungs, also in other bones (skull, long bones), and occasionally in lymphatic glands and in the spleen. The prognosis is of the utmost gravity, the majority of cases die in spite of treatment within a period of three years.

\section{Ewing's Tumour.}

First described by Ewing in I92I under the heading of "Endethelioma," it forms about I5 per cent. of malignant primary tumours of bone. Most cases occur before the age of twenty-one, the commonest age incidence being between five and fifteen years of age, somewhat earlier than osteogenic sarcoma. Males are affected twice as frequently as females. The commonest bone affected is the tibia, and in order of frequency the following bones are involved : tibia, fibula, humerus, femur, jaws. The lower limb, as in the case of osteogenic sarcoma, is still the common site, but the bones most frequently affected are not the same. Simultaneous appearance of tumours in two contiguous bones, f.i., tibia and fibula, is not very uncommon. The relative incidence of Ewing's tumour in small bones and the jaws is also much greater than in the osteogenic variety. In long bones the central portion of the diaphysis is involved more frequently than the metaphyseal ends. Bone destruction is slight, new bone formation and osteosclerosis less obvious and less characteristic than in osteogenic sarcoma. The histological appearance of the tumour is a uniform mass of round cells with clear scanty cytoplasm containing small darkly staining nuclei; there is a characteristic absence of intercellular material. The clinical manifestations are pain, tumour and fever. Pain is intermittent ; it increases both in intensity and frequency as the disease progresses. Local tenderness and pain on pressure are common. Fever varies from $100^{\circ}$ to $103^{\circ} \mathrm{F}$., or even higher ; it is present in about one-third of the cases early in the history and is found in most cases at the later stages. The association of pain, fever, local tenderness and the difficulty in the interpretation of radiological appearances not infrequently lead to a diagnosis of osteomyelitis. The appearance of the tumour follows pain in most cases. The swelling is fusiform in the long bones, round or oval in the small bones and jaws ; it is soft and elastic in consistency and tender on palpation. The course of the disease often shows periods of spontaneous quiescence in between periods of activity. Most cases are of great virulence, some running a course of six to nine months only. others surviving up to two years. The tumour is highly malignant and gives rise to metastasis in the skull, lymph glands, lungs, subcutaneous tissue and viscera. In about one-third of the cases the prognosis, although always uncertain, is somewhat more favourable.

\section{Multiple Myeloma.}

As implied by its name, the main clinical characteristic of this type of tumour is the multiplicity of original foci. It is a neoplasm affecting the bone marrow and is the rarest of bone tumours. The age incidence is between the fourth and sixth decades, and it is twice as common in men than in women. In order of frequency the bones affected are: ribs, vertebræ, sternum, skull, innominate bone, and clavicle. The simultaneous appearance of multiple tumours is characteristic of the disease; there are, however, on record a few proved cases where only a solitary tumour was detected even on post-mortem examination; this rare type of single myeloma is of better prognosis. The tumours have great osteolytic power and destruction of bone without any compensatory new bone formation is the rule; spontaneous fracture is common. Histologically the bulk of the tumour is composed of plasma cells, but other bone marrow cells, lymphocytes, myelocytes and even erythrocytes are also found and in some tumours may form the main part of the cellular elements; the stroma is scanty and the lack of intercellular material and the eccentric nucleus of the plasma cell all give a clear histological picture. The clinical course is that of a progressive malignant tumour of insidious onset, characterised by pain in the affected bones, general malaise, lassitude, weakness and loss of weight. The presence of a tumour is usually detected clinically first in the ribs, the clavicle or in the iliac bone; in the vertebræ or other portions of the innominate bone only radiological examination may reveal the presence of a neoplasm. Examination of the urine reveals the presence of Bence-Jones albumose in about half the number of cases except in the late or terminal stages, when nearly all cases show this abnormality in the urine. In the fully established disease, anæmia is a marked feature. Metastasis are found in the liver and spleen. 


\section{FIG. 1.}

FIG. I.-A. Osteogenic Sarcoma of mandible-_" periosteal " type showing " sun-ray " spicules.

B. Osteogenic Sarcoma of scapula osteolytic type.

C. Osteogenic Sarcoma of femur. Osteoblastic type.

A FIG. II.

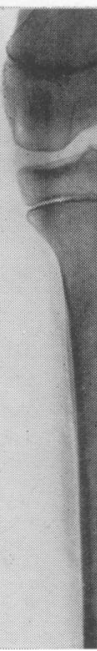

FIG. II.-A and B. Ewing's tumour involving Tibia and Fibula.

Proved by biopsy. Multiple metastasis and death
within twelve months. A. Antero-posterior view.

Proved by biopsy. Multiple metastasis and death
within twelve months. A. Antero-posterior view. B. Lateral view.

B

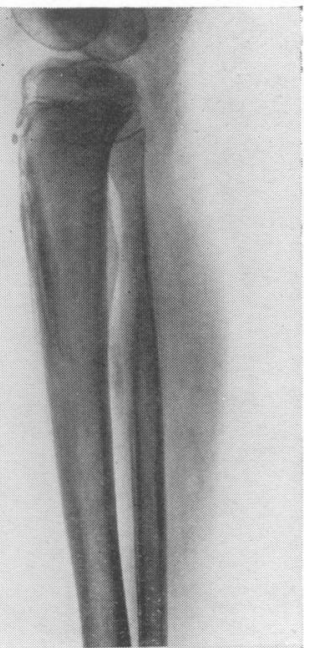

A FIG. III.

B

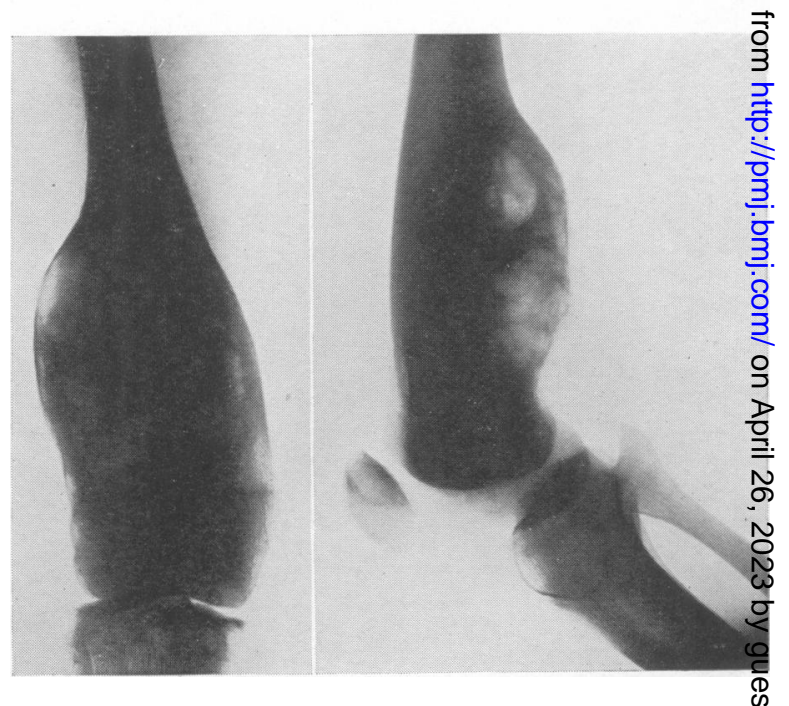

FIG. III.-A and B. Osteoclastoma of femur. Five years after treatment by conservative surgery. Clinically $\$$ normal limb. A. Antero-posterior viewo B. Lateral view. 


\section{Parosteal Sarcoma.}

This tumour, primarily derived from the soft tissues in close apposition to the boneperiosteum and capsular and ligamentous structures, invades the bone from without. It consists of fusiform cells, similar to the fibrosarcoma of tendons and tendon sheaths, with additional cartilagenous and myxomatous elements, some of which undergo ossification. Most cases occur in adults, the peak age incidence is thirty years; the sex incidence is equal. In some respects this type resembles osteogenic sarcoma, thus the most common bone affected is again the femur, and in order of frequency the bones involved are : femur, tibia, innominate bone, scapula, humerus, skull, radius and ulna.

The clinical course, however, is longer; tumour formation precedes the appearance of pain, which is never as severe as in osteogenic sarcoma or Ewing's tumour, unless from direct involvement of nerves. The tumour invades the bone and the surrounding soft tissues; it is usually bulky, lobulated and well delimited. Metastasis take longer to appear than in other types; the usual site is in the lungs.

\section{Osteoclastoma.}

This tumour, also known under the name of giant-cell tumour, accounts for 25 per cent. of all bone tumours. The common age incidence is between sixteen and thirty years, but, as in the case of other bone neoplasms, it may be found at any age, in young and old alike. Men and women are affected with equal frequency. The commonest site is the femur; in order of frequency the bones affected are : femur, tibia, radius, jaw, humerus, innominate bone. An interesting feature is that although the region of the knee still accounts for the majority of cases, there is a sex variation in its incidence, the lower end of the femur being usually affected in men and the upper end of the tibia in women. Histologically the main characteristic feature is the presence, generally in abundance, of multinicleated giant-cells, resembling normal osteoclasts and containing numerous nuclei equal in size and centrally situated. The tumour is very vascular, causing progressive bone absorption, leading eventually, if untreated, to spontaneous fracture. Clinically there is nearly always a history of trauma, followed after a varying interval by the development of pain and the appearance of a tumour. The X-ray appearances (see below) are very typical and establish the diagnosis with certainty in most cases. In the vast majority of cases the disease pursues a benign course. In 5 per cent. of patients malignant changes are found, either ab initio, in the primary tumour, or subsequently, generally following inadequate or ill-advised treatment; or silently by the appearance of metastasis, generally single, occasionally multiple, and nearly always in the lungs.

\section{RADIOLOGIGAL APPEARANGES.}

The radiological findings in cases of primary malignant tumours of bone are extremely important, but their usefulness depends firstly on faultless technique and secondly on the experience and good judgment of the radiologist. Some features are so characteristic as to leave no room for doubt as to the type of neoplasm ; but other appearances present difficulty in interpretation, not only as to the type of tumour, but also in the distinction between inflammatory conditions and neoplasms and in the differentiation between primary and metastatic tumours. It must be emphasised that, however important, radiological findings are only aids to diagnosis and should be taken together with the clinical findings and in the light of all the other means of investigation. It is, nevertheless, so important a part of the examination as to warrant a description of the main characteristics in each of the five types.

Osteogenic Sarcoma (Fig. I). The typical radiographic appearances are as follows : (I) Bone destruction : multiple areas of irregular localised rarefaction involving both cortex and medulla, associated with areas of sclerosis. (2) Bone formation : One of the following may be present: (a) the "Sun-ray " spicules, at right-angle to the main axis of the bone ; $(b)$ Codman's triangle, a wedge-shaped subperiosteal new bone formation at the periphery of the tumour ; $(c)$ longitudinal new bone formation; $(d)$ irregular masses of new bone. Any one of these features may be present alone or in combination. Osteosclerosis, peripheral new bone and osteolysis are all to be found in varying degrees in the same tumour.

Ewing's Tumour (Fig. 2). Here the X-ray findings, specially in the early stages, are often misleading. The more usual features are $(a)$ increased density of the shaft involving both cortex and medulla; $(b)$ absorption of the shaft with widening of the medullary cavity; (c) new periosteal bone deposited along the long axis of the bone in successive lamellæ, the 
so-called " onion-peel " appearance ; $(d)$ patchy absorption of cortical bone with a deposit of ostheophyte-like masses in the central part of the diaphysis simulating osteomyelitis.

Multiple Myeloma. Bone destruction without any compensatory new bone formation is the characteristic feature. Portions of the bones involved may appear to be completely absent. The affected areas are of uniform density, without trabeculations in the centre or sclerosis at the periphery. The appearances resemble closely those seen in some types of carcinomatous metastasis.

Parosteal Sarcoma. In this type there is erosion of bone from outside, in the early stages ; later osteolysis and sometimes irregular calcification in the soft tissue part of the tumour.

Osteoclastoma. The common radiological characteristics are: thinning of the cortex, expansion of bone, absence of subperiosteal new bone formation and trabeculations in the osteolytic area. A rarer variety is an area of complete bone absorption, without expansion of trabeculation closely resembling a cyst.

\section{BIOPSY.}

The question of biopsy still remains a controversial point. It has its strong advocates and equally fierce opponents. The following is a summary of the author's personal views :-

Osteogenic Sarcoma. A biopsy increases or hastens the risks of dissemination. If the diagnosis is not in doubt, biopsy is not necessary; if in doubt, it should be done with the diathermy, and under a tourniquet. In any case, it should only be undertaken if amputation is contemplated. Biopsy is of no benefit to the patient if radiological treatment alone is to be given.

Ewing's Tumour. As this tumour is highly radiosensitive, a preliminary test radiation should always precede biopsy. A small tumour dose generally suffices to produce rapid and marked regression of the tumour, so establishing a diagnosis and rendering biopsy unnecessary. If the lesion, presumed to be a Ewing's tumour does not respond to a test radiation, it is most probably not a Ewing's tumour and biopsy should be performed.

Multiple Myeloma. Aspiration biopsy by sternal puncture or by puncture of any accessible tumour is the method of choice. It should be done in all cases where there is a doubt as to the nature of the tumour. There is no risk in the procedure as the tumour is multiple $a b$ initio, and the alternative diagnosis is as a rule dissemination from a carcinoma clinically silent.

Parosteal Sarcoma. Biopsy is justifiable ; there is no risk of dissemination as far as can be judged from the natural history of these tumours. Nevertheless, a tourniquet should be applied whenever possible and the section taken with the diathermy knife.

Osteoclastoma. Biopsy is harmless as the tumour is benign; if carried out, curetting of the entire tumour, if possible, should be done.

\section{TREATMENT.}

The methods of treatment available to the victim of a primary malignant tumour of bone are few and consist of radical surgery, radiation by X-rays or radium, or a combination of both methods. If a successful result is to be obtained, the decision as regards the method should be taken without bias or prejudice for or against any particular method of therapy. It should never be dictated solely or mainly by the available facilities, but by experience based on the study of and results obtained by various methods and guided by the natural history of the particular tumour. There is, unfortunately, no universal agreement as to the best procedure in every case, mainly because the end results of treatment by any method are still extremely bad. Surgery, even in the best of hands and with the greatest skill and courage, has achieved little at the expense of great risk and grave mutilation. Radiation, in spite of rapid progress both in the apparatus provided and in the technique used, still holds more in its promise than in actual achievement. Other methods of treatment, toxins, various metals (gold, lead, selenium, etc.) should not even enter into consideration as a primary or sole method of therapy. From the study of over a hundred cases and from the assessment of end results obtained in Westminster and Mount Vernon Hospitals, the author formed the following conclusions, which, of necessity, are not final, but represent his views in the light of experience to date.

Osteogenic Sarcoma. In view of the paucity of results by surgery as the sole method of treatment, radiation should be the initial treatment. But as the results of radiation, even when complete regression of the growth has been obtained, offer no security as to the permanent eradication of the disease, radiation followed by amputation should be considered. The cases 
can be divided into two groups : (I) operable cases in adults ; (2) inoperable cases in adults and all cases in children, regardless of operability. In the first group, radiation is a preoperative measure, it should aim at the delivery of the maximum desirable tissue dose, such as 8,000 to ro,ooor in a period of three to four weeks ; this to be followed within two to three weeks by radical surgical treatment. In the second group of cases, no surgical treatment is contemplated, and the radiation treatment should be by the protracted, fractional method delivering an adequate total tissue dose in a period of five to six weeks. This causes less subsequent damage to normal tissues (skin, tendons, bones and periarticular tissues), and permits further radiation in six to eight months should the need for it arise. Repeated periodical examination, clinically and radiologically, show the effect of treatment ; regression of the tumour and restoration of the bone to a normal condition may continue for several months. Should regression cease or recrudescence of activity be noticed, further treatment can be given or surgery decided upon. In inoperable cases the method is obviously radiation ; in operable cases in adults amputation should be considered. In children amputation is only indicated for the relief of intractable pain otherwise uncontrolled. Prophylactic radiation of the lungs has proved a failure, and as long as no metastasis are detected, and even if present but clinically silent, radiation should be delayed.

Ewing's Tumour. This is the most radiosensitive of all bone neoplasms; the response to radiation is rapid and disappearance of large tumour masses and restoration of the affected bone to a normal condition occurs in the majority of cases. Permanent regression, nevertheless, is but seldom achieved. Local recurrences occur, but seldom after an adequate treatment, but multiple metastasis are the common final manifestation. In the event of local recurrence in the absence of metastasis, amputation is indicated. But the primary surgical treatment of a Ewing's tumour is as little justifiable as the excision of lymph glands in lymphosarcoma.

Multiple Myeloma. The disease is invariably fatal; it does not lend itself to surgical treatment owing to the situation and multiplicity of lesions. Pain is the most frequent and the most distressing symptom ; radiation in small doses is the best palliative remedy.

Parosteal Sarcoma. These tumours are radioresistant. Large tissue dosage is required to produce an effect on the tumour. In a limb, specially in the vicinity of a joint, such treatment is apt to be followed by marked deformity, loss of function, a prolonged period of incapacity and eventually an uneconomical limb. Surgical treatment is therefore the method of choice in all operable cases. Post-operative radiation to the stump is of value. Palliative irradiation in inoperable cases should be given with caution.

Osteoclastoma. Good results have been obtained both by conservative surgery (Fig. 3) and by radiation. The choice of method depends upon the following factors : site of tumour, its size and the amount of bone destroyed by it ; the age and general condition of the patient. Surgery is indicated in preference to irradiation (I) in young children, when the tumour is in close proximity to the epiphyseal line and radiation may be followed by arrest of growth at the growing end of a long bone; (2) in tumours of moderate extent, when the destruction of bone is limited and the cavity left by curetting small ; (3) in tumours of the digits ; (4) when the tumour has extended to the neighbouring joint and irradiation will have to include articular and peri-articular structures. Irradiation is indicated (I) in healthy adults ; (2) in all patients where the tumour is difficult of access, such as the innominate bone ; (3) in accessible tumours when the destruction of bone is great and pathological fracture likely to follow curetting. Complete recalcification has been obtained by radiation alone, and the total dose required is small-in the region of 3,00or. The effects of radiation become apparent within six weeks of the treatment and continue for eight to ten months. It is generally agreed that the combination of treatment by curetting and radiation is not advisable. If the tumour is completely removed by scraping, radiation is not needed, as it does not hasten repair ; it may even delay it by its effect on the newly-formed capillary vessels. In the case of a malignant giant-cell tumour, radiation following a biopsy is a more rational procedure. Radiation of post-operative recurrences is less successful than radiation as the sole method of treatment. Radiation should always be given by X-rays or teleradium; interstitial radiumtherapy is dangerous, as it has been known to transform an osteoclastoma into a highly malignant osteogenic tumour.

I am indebted to Air Marshal Sir Harold Whittingham, Director General, Medical Services, Royal Air Force, for permission to reproduce Fig. 3 . 